\title{
MISMATCH IN PHOTODIODE AND PHOTOTRANSISTOR ARRAYS
}

\author{
Zaven K. Kalayjian and Andreas G. Andreou \\ Johns Hopkins University \\ Department of Electrical and Computer Engineering \\ Baltimore, MD 21211
}

\begin{abstract}
We characterized photodetector mismatch in $2 \mu \mathrm{m}$ and $1.2 \mu \mathrm{m}$ CMOS processes. $32 \times 32$ element photodiode and phototransistor arrays were fabricated in each process. Light response measurements were made using a DC light source and neutral density filters. Dark currents were also measured and characterized. Our measurements reveal less than $2 \%$ mismatch for photodiodes over 4 orders of magnitude of intensity, and less than $5 \%$ mismatch for phototransistors. The oxide profile above the photodetector array is shown to be responsible for edge-effects.
\end{abstract}

\section{INTRODUCTION}

In this paper, we investigate mismatch in integrated silicon photodetector arrays. Photodiode and phototransistor arrays have been used in many VLSI imaging and image processing systems.[2, 4] While CMOS imagers typically have fixed pattern noise (FPN) correction circuitry on-board to cancel offsets introduced by transistor threshold mismatch during pixel readout, image processing arrays typically operate directly on photodetector photocurrents. $[3,1]$ We studied the matching properties of photodiode and phototransistor arrays fabricated in $2 \mu \mathrm{m}$ and $1.2 \mu \mathrm{m} \mathrm{n}$-well CMOS processes to determine the magnitude of non-uniformity in the photocurrents.

\section{DARK CURRENT AND PHOTOCURRENT MODELS}

Current from junction photodetectors can be separated into two components: dark current and photocurrent.

Current measured from a photodiode or phototransistor in the absence of light, known as dark current, arises from thermally generated carriers that are caught in the depletion region of the photodetector. Dark current is identical to the reverse bias leakage current in a diode, and is described by the following equation:

$$
I_{\text {dark }}=-q A \beta\left(p_{n} \frac{L_{p}}{\tau_{p}}+n_{p} \frac{L_{n}}{\tau_{n}}\right)
$$

where $A$ is the area of the junction, $p_{n}$ and $n_{p}$ are minority carrier concentrations, and $L$ and $\tau$ are the minority carrier diffusion length and lifetime, respectively. The factor $\beta$ is the current gain for phototransistors; $\beta=1$ for photodiodes.

In an array of photodetectors, mismatch in dark currents will be caused by the following: 1) Lithographic variability, which will affect junction cross-section $A, 2$ ) Variations in doping concentration, which will affect diffusion length, carrier lifetime, and $\beta$. In this analysis, we assume that variations in lithography or doping uniformity are normally distributed.
Photogenerated carriers are indistinct from thermally generated carriers. Therefore, we model photocurrent using an equation similar to the one above:

$$
I_{\text {photo }}=-q A \beta\left(p_{\text {opt }} \frac{L_{p}}{\tau_{p}}+n_{\text {opt }} \frac{L_{n}}{\tau_{n}}\right)
$$

where $p_{\text {opt }}$ and $n_{\text {opt }}$ are the concentration of photogenerated free carriers. In general, $p_{o p t}=n_{o p t}$ and will be proportional to the photon flux $\phi$. We relate photocurrent to incident light flux:

$$
I_{\text {photo }}=-q A \beta \eta \phi
$$

where photo-generation of carriers and carrier capture by the depletion region is summarized in $\eta$, the quantum efficiency.

The total photocurrent is proportional to the sum of the dark current and photogenerated currents:

$$
I_{\text {total }}=-q A \beta \eta \phi+I_{\text {dark }}
$$

Under conditions of high illumination, the contribution of $I_{d a r k}$ can be made very small in proportion to $I_{\text {photo }}$. Consequently, we can examine the matching properties of each term in the above equation separately. From Equations 1 and 2, we deduce that the same processes that give rise to mismatch in dark current will cause the light response to be mismatched.

\section{EXPERIMENTAL SETUP AND PROCEDURE}

We subjected the photodetector arrays to a uniformly intense field of light and measured the current output from each pixel in the array. Measurements at various intensities were made to determine if variations in uniformity were caused by gain or offset mismatch in the photodetectors. Additional measurements were made in complete darkness to investigate leakage currents throughout the array. Temperature was monitored during measurements.

We measured the photocurrents from 8 separate $32 \times 32$ arrays of photodetectors fabricated in two CMOS technologies: $2 \mu \mathrm{m} \mathrm{n}$ well and $1.2 \mu \mathrm{m} n$-well. In both technologies, photodiodes were fabricated using $\mathrm{n}+$ diffusion in $\mathrm{p}$-type substrate, and phototransistors used a p+ diffusion emitter, n-well base, and p-type substrate collector. Photodetectors were fabricated with a $50 \lambda$ pitch in both process. By keeping the same dimensions in $\lambda$ between processes, photodetectors from the $1.2 \mu \mathrm{m}$ technology were smaller in physical dimensions than in the $2 \mu \mathrm{m}$ technology.

Random access multiplexers at the periphery of the array allowed $x-y$ addressing of any one pixel at a time. During readout, the voltage across a selected photodetector was clamped to 


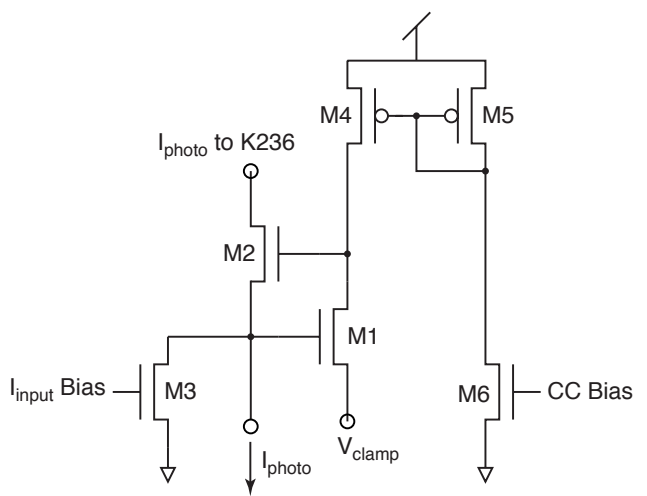

Figure 1: During readout, a two-transistor current-conveyor ( $M 1$ and $M 2$ ) clamps the voltage across the photodetector to a programmable voltage $V_{\text {clamp }}+V_{T_{M 1}}$. $M 2$ conveys the photocurrent $I_{\text {photo }}$ to an off-chip ammeter.

a programmable voltage by an on-board current-conveyor readout circuit (Figure 1). This ensured that all photodetector currents in the array were produced under equivalent bias conditions. The current-conveyor circuit also provided a high-impedance node from which the small photocurrents were measured.

Photocurrents were measured using a computer controlled Keithly 236 Source/Measure Unit (SMU). A Macintosh computer with a National Instruments data acquisition card was used to automatically scan through the photodetectors in an array. Photocurrents were recorded by the computer through a general purpose interface bus (GPIB) connection to the SMU.

The experimental setup, shown in Figure 2, used an incandescent DC light source at a distance of $20 \mathrm{~cm}$ to illuminate the array being measured. Incident light intensity was varied by interposing neutral density filters between the light source and the photodetector array. A wire-wrap board provided power and instrumentation connections to the array being measured.

The experimental procedure for testing uniformity follows:

1. The DC light source was powered and allowed to thermally stabilize.

2. A 40-pin DIP packaged array was selected, loaded into the wire-wrap board and allowed a few minutes to reach thermal equilibrium with the test system.

3. With the chip die exposed to direct light from the DC source, the array was sequentially scanned, row by row, and the measured photocurrents recorded. This constituted the no-filter (NF) measurement.

4. A neutral density (ND) filter was placed over the die. (The ND filter contacted only the package, and not the surface of the die.) The system was allowed to reach thermal equilibrium, and a new set of photocurrent measurements were recorded.

5. The ND filter was then swapped for one with a lower transmission coefficient, and the above step was repeated. Each successive ND filter had a lower transmission coefficient.

6. The last measurement made used an opaque covering over the array. This final step constituted the dark current (Dark) measurement.

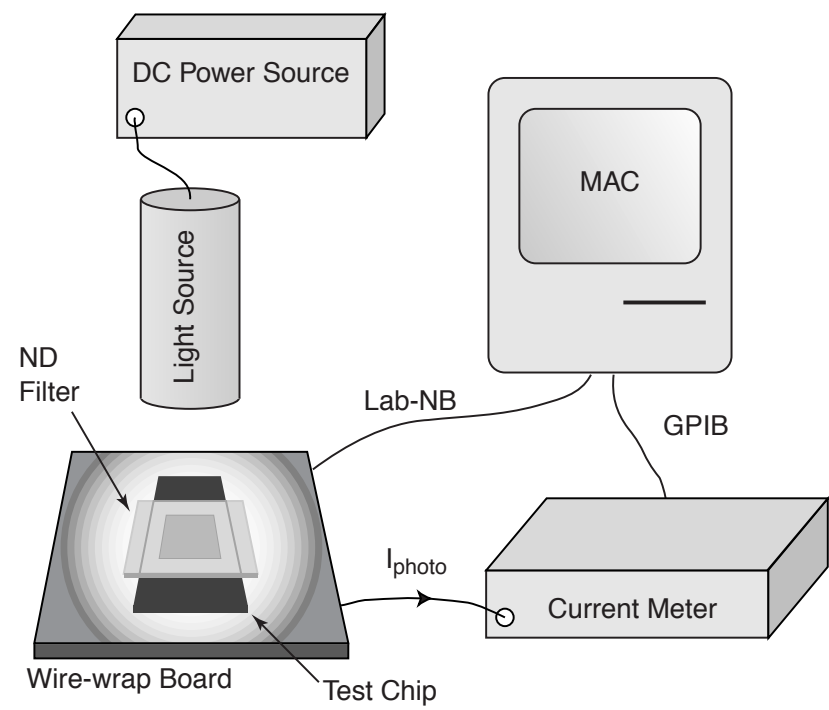

Figure 2: The computer controlled data acquisition system for testing uniformity of photodetector arrays used a Lab-NB interface to scan through the photodetector array, a DC light source, and a GPIB controlled current meter

Figure 3 shows data from two of the eight photodetector arrays tested - one phototransistor array from the $1.2 \mu \mathrm{m}$ process and one photodiode array from the $2 \mu \mathrm{m}$ process. In the figure, a map of the measured photodetector currents as a function of coordinate is displayed under two illumination conditions: NF (no ND filter interposed between light source and chip), and Dark (opaque cover slip interposed between light source and chip). Other measurements were taken using various combinations of ND filters, and are used in later analysis. Note that light responses from arrays fabricated in the two technologies are not directly comparable, as measurements were made under slightly different experimental conditions (different light intensity). Dark current and light response are analyzed in the following sections.

\section{DARK CURRENTS}

In the preceding section, we postulated that variations in junction area and doping density would be normally distributed. To assess if systematic or experimental conditions affected photocurrent measurements, and to see how well the above assumptions correspond to our measurements, we compare the cumulative distribution function (CDF) of the data to an ideal CDF for a Gaussian random variable using a quantile-quantile (QQ) plot. The purpose of the QQ plot is to qualitatively determine whether the measured data can be modeled using a Gaussian random variable. If the samples come from a Gaussian distribution, the plot will be linear.

Figure 4 shows histograms of the raw dark current data for the photocurrents plotted in Figure 3 and the ideal and measured CDF in a QQ plot. The distribution of measured dark currents for the $1.2 \mu \mathrm{m}$ detector array conforms well to the assumption of normally distributed variations in photodetector dark current. The Dark photocurrent map shown in Figure 3 for the $1.2 \mu$ m process shows a random distribution of photocurrents, which the qq plot qualitatively confirms to be normally distributed. On the other 
$1.2 \mu \mathrm{m}$
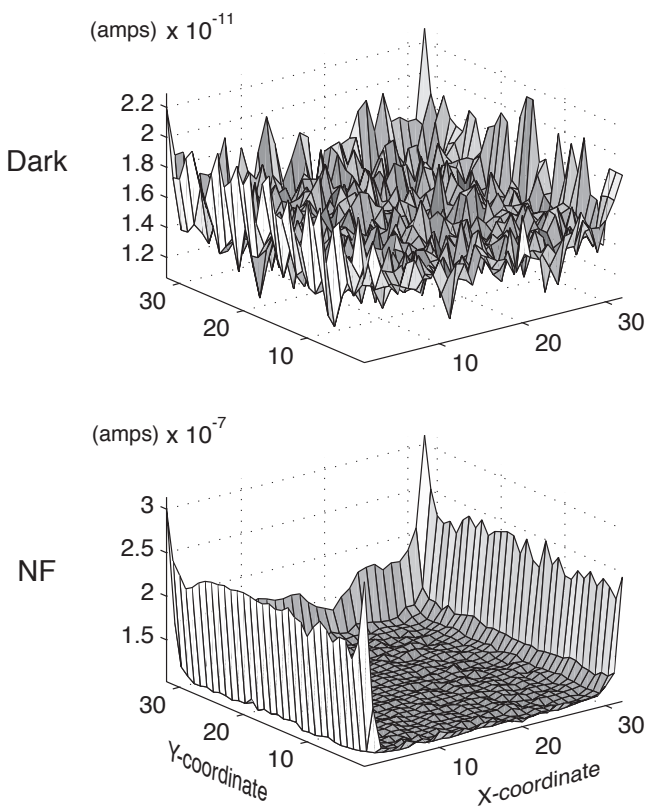

$2 \mu \mathrm{m}$
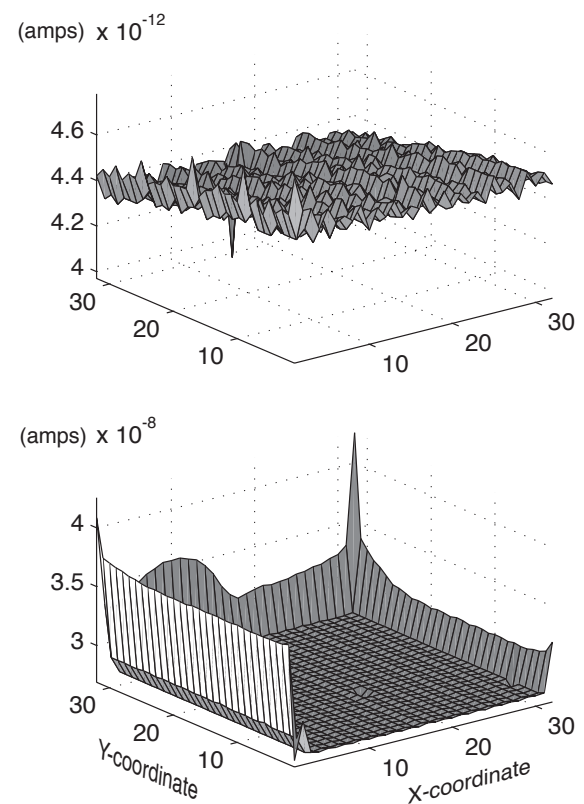

Figure 3: Dark and NF measured data as a function of coordinate position for phototransistor (left) and photodiode (right) arrays in $1.2 \mu \mathrm{m}$ and $2 \mu \mathrm{m}$ processes.

hand, there is a significant deviation in the QQ plot of the $2 \mu \mathrm{m}$ detector array. A gradual drift in photocurrents in the $2 \mu \mathrm{m}$ array indicates that the experimental setup had not reached thermal equilibrium. This statistical analysis was performed on the Dark measurements for the 8 photodetector arrays.

Table 1 shows the statistics computed for the dark current data for all measured photodetector arrays. Those arrays whose QQ plots show data affected by temperature drift are italicized and included for reference. Some of the measurements that did evidence temperature effects nevertheless showed tight grouping around the mean in their histograms, reflected by a low Fano factor $\sigma / \mu$. Although the $2 \mu \mathrm{m}$ photodetectors had larger geometries, the $1.2 \mu \mathrm{m}$ photodetectors had larger dark currents. This is due to higher doping concentrations in the smaller-featured process which would contribute to a higher concentration of recombination-generation centers in the semiconductor.[5] Consequently, area is less important a factor for photodetectors under dark conditions than bulk crystalline properties.

We expected phototransistors to have higher dark current levels than photodiodes due to internal current gain. However, while the $1.2 \mu \mathrm{m}$ phototransistors did have higher leakage than the $1.2 \mu \mathrm{m}$ photodiodes, the $2 \mu \mathrm{m}$ phototransistors inexplicably had lower leakage current levels than the $2 \mu \mathrm{m}$ photodiodes. Overall, dark currents varied by around $1 \%$ around the mean value in the photodiode arrays tested, around $5 \%$ for the photodetectors.

\section{LIGHT RESPONSE}

Eleven measurements using different ND filters were made for photodetector and photodiode arrays. The measurements span four orders of magnitude of illumination intensity. Both photodiodes and phototransistors responded linearly to changes in illumination intensity. Phototransistor gain in $1.2 \mu \mathrm{m}$ and $2 \mu \mathrm{m}$ processes was measured to be approximately 50 and 25 , respectively.
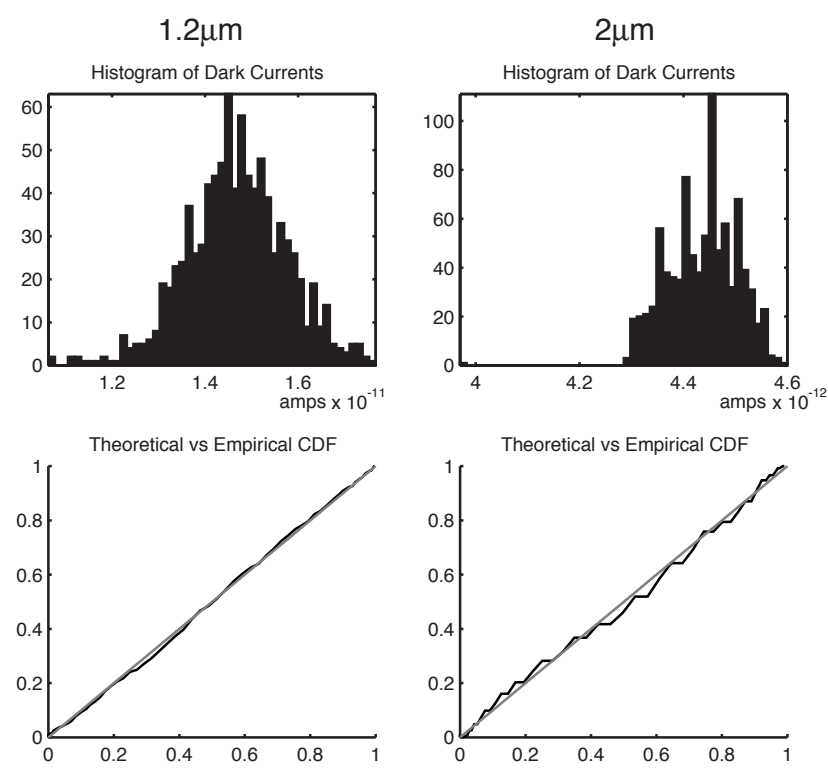

Figure 4: Dark current histograms and quantile-quantile plots for Dark conditions. QQ plots were used to verify our assumption that variability in dark current is normally distributed. 


\begin{tabular}{|c|c|c|c|c|c|c|c|c|}
\hline$I_{\text {dark }}$ Stats & \multicolumn{4}{|c|}{$1.2 \mu \mathrm{m}$} & \multicolumn{4}{|c|}{$2 \mu \mathrm{m}$} \\
\hline & pd1 & pd3 & pt3 & pt5 & pd2 & pd3 & pt1 & pt4 \\
\hline$\mu(\mathrm{pA})$ & 9.77 & 8.69 & 18.22 & 13.46 & 4.07 & 4.44 & 3.33 & 2.37 \\
\hline$\sigma(\mathrm{pA})$ & 0.182 & 0.122 & 0.856 & 0.788 & 0.302 & 0.066 & 0.447 & 0.0529 \\
\hline$\sigma / \mu$ & 0.019 & 0.014 & 0.047 & 0.059 & 0.074 & 0.015 & 0.13 & 0.022 \\
\hline
\end{tabular}

Table 1: Dark current statistics for photodetector arrays. $\mathrm{pd}=$ photodiode and $\mathrm{pt}=$ phototransistor array. Measurements affected by temperature drift are marked with italics.

\begin{tabular}{|c|c|c|c|c|c|c|c|c|}
\hline & \multicolumn{4}{|c|}{$1.2 \mu \mathrm{m}$} & \multicolumn{4}{|c|}{$2 \mu \mathrm{m}$} \\
\hline Gain Stats & pd1 & pd3 & pt3 & pt5 & pd1 & pd3 & pt3 & pt5 \\
\hline$\mu(\mathrm{nA})$ & 2.16 & 2.06 & 109 & 114 & 27.8 & 27.5 & 608 & 601 \\
\hline$\sigma(\mathrm{nA})$ & 0.0437 & 0.0343 & 4.66 & 7.78 & 0.0787 & 0.0691 & 7.67 & 7.19 \\
\hline$\sigma / \mu$ & 0.0203 & 0.0167 & 0.0429 & 0.0682 & $2.83 \mathrm{e}-3$ & $2.51 \mathrm{e}-3$ & 0.0126 & 0.0120 \\
\hline Offset Stats & & & & & & & & \\
\hline$\mu(\mathrm{pA})$ & -1.09 & 1.45 & -10.2 & -21.2 & 0.635 & -0.133 & 21.1 & 20.3 \\
\hline$\sigma(\mathrm{pA})$ & 1.05 & 0.956 & 108 & 119 & 7.46 & 2.25 & 268 & 232 \\
\hline$\sigma / \mu$ & -0.970 & 0.658 & -10.6 & -5.61 & 11.8 & -170 & 14.4 & 8.08 \\
\hline
\end{tabular}

Table 2: Light response statistics for $\mathrm{pd}=$ photodiode and $\mathrm{pt}=$ phototransistor arrays. Gain and Offset were computed using Equation 4.

The NF data in Figure 3 shows a phenomenon common to all illuminated photodetector arrays: a pronounced edge-effect, which is manifest across both technologies and for both photodiodes and phototransistor array. The edge-patterns differ between technologies. In the $1.2 \mu \mathrm{m}$ process, the edge-effect appears to extend further into the array than for the $2 \mu \mathrm{m}$ process. This edge-effect gradually decays with decreasing illumination, and is barely noticeable under Dark conditions. For this reason, we propose that oxide non-uniformities are responsible for these edge-effects.

Using Equation 4, we performed linear regression on the eleven intensity versus photocurrent data points for each detector in the arrays. Table 2 shows the gain $(-q A \beta \eta \phi$ term) and offset ( $I_{\text {dark }}$ term) statistics for the photodetector arrays in each process. The mean offset values slightly undershoot physically expected zero-intensity current response, but all are within a few $\sigma$ of zero current at zero intensity. The variation in offset is quite large, with the standard deviation exceeding the mean in some cases. However, the effect of these offset variations is quite small compared to the photocurrent of an illuminated array.

QQ plots for the gain parameter (not shown here) indicates normally distributed variability. We observe that the Fano factors for the gain of the photodetectors is much lower than those for dark currents. In the presence of light, the majority of the current arises from captured photogenerated carriers. Thus, the main source of variation in the data will be from the light dependant term in Equation 2. This term depends on area in photodiodes, and on area and gain in phototransistors. Since photodetector area is lithographically defined, we can conclude that light response variability of photodiodes is mostly due to lithographic mismatches. In the $1.2 \mu \mathrm{m}$ process, photodiodes areas are matched to approximately a $2 \%$ tolerance; in the $2 \mu \mathrm{m}$ process, $0.3 \%$.

\section{CONCLUSIONS}

We have measured the photocurrents from integrated photodiode and phototransistor arrays in two different CMOS processes. Dark currents and light response over four orders of magnitude of illu- mination were measured for eight photodetector arrays. We assumed that normally distributed lithographic variations and doping concentration non-uniformities would contribute to photodetector variability and that these variations would similarly affect both dark currents and light response.

Our data confirms that the variability in dark current and light response is closely matched. In particular, the Fano factor for photodetector gain, extracted from illumination versus intensity data, matches the Fano factor for the measured dark current statistics. Significant edge-effects under illuminated conditions were observed in the photocurrents of all arrays, but were absent in dark current measurements. We conclude that these edge effects are induced by oxide irregularities at the periphery of the array.

Phototransistors were found to be more poorly matched than photodiodes. We conclude that doping variations which contribute to photodiode matching are compounded in phototransistors due to the intrinsic gain of these devices.

\section{REFERENCES}

[1] A. G. Andreou and K. A. Boahen. A 48,000 pixel, 590,000 transistor silicon retina in current-mode subthreshold cmos. Proc. 37th Midwest Sympsium on Circuits and Systems, 1989.

[2] T. Delbrück and C. Mead. Analog VLSI phototransduction. CNS Memo 30, California Institute of Technology, Pasadena, California, May 1994.

[3] Z. Kalayjian and A. Andreou. Integrated high-resolution focal-plane polarization imager using polymer micropolarizers. Proc. 2nd Int. Workshop on Mixed-mode Int. Circ. and App., 1998.

[4] D. Scheffer, B. Dierickx, and B. Meynants. Random addressable 2048x2048 active pixel image sensor. IEEE Trans. on Elec. Dev., 44(10):1716-1720, 1997.

[5] H. Wong. Technology and device scaling considerations for CMOS imagers. IEEE Tran. on Elec. Dev., 43(12):21312142, 1996. 